\title{
FUNCTION SPACES OF COMPLETELY METRIZABLE SPACES
}

\author{
JAN BAARS, JOOST DE GROOT, AND JAN PELANT
}

\begin{abstract}
Let $X$ and $Y$ be metric spaces and let $\phi: C_{p}(X) \rightarrow C_{p}(Y)$ (resp. $\left.\phi: C_{p}^{*}(X) \rightarrow C_{p}^{*}(Y)\right)$ be a continuous linear surjection. We prove that $Y$ is completely metrizable whenever $X$ is. As a corollary we obtain that complete metrizability is preserved by $l_{p}$ (resp. $l_{p}^{*}$-equivalence) in the class of all metric spaces. This solves Problem 35 in [2] (raised by Arhangel'skii).
\end{abstract}

\section{INTRODUCTION}

Let $X$ and $Y$ be metric spaces. By $C(X)$ (resp. $C^{*}(X)$ ), we denote the set of all real-valued continuous functions (resp. the set of all real-valued bounded continuous functions), on $X$. We endow $C(X)$ (resp. $C^{*}(X)$ ) with the topology of pointwise convergence and denote that by $C_{p}(x)$ (resp. $\left.C_{p}^{*}(x)\right)$. We define $X$ and $Y$ to be $l_{p}$-equivalent (resp. $l_{p}^{*}$-equivalent) whenever $C_{p}(X)$ and $C_{p}(Y)$ (resp. $C_{p}^{*}(X)$ and $\left.C_{p}^{*}(Y)\right)$ are linearly homeomorphic. A topological property $\mathscr{P}$ is said to be preserved by $l_{p}$-equivalence (resp. $l_{p}^{*}$-equivalence) whenever for $l_{p}$-equivalent (resp. $l_{p}^{*}$-equivalent) spaces $X$ and $Y$ we have that $X$ has property $\mathscr{P}$ if and only if $Y$ has property $\mathscr{P}$.

In [2, Problem 35], Arhangel'skii asks whether complete metrizability is preserved by $l_{p}$-equivalence for the class of all separable metrizable spaces. In this paper we answer this question in the affirmative, even for arbitrary metric spaces. In fact we prove for metric spaces $X$ and $Y$ and for a continuous linear surjection $\phi: C_{p}(X) \rightarrow C_{p}(Y)$ that $Y$ is completely metrizable whenever $X$ is. Moreover we obtain the same result for $l_{p}^{*}$-equivalence. The assumption that the mappings between function spaces are linear is essential. In [10], Dobrowolski, Gul'ko, and Mogilski proved that for any two nondiscrete countable metric spaces $X$ and $Y, C_{p}(X)$ and $C_{p}(Y)$ are homeomorphic. In particular this holds for the space of rationals $\mathbb{Q}$ and a convergent sequence.

\section{Preliminaries}

Let $X$ and $Y$ be metric spaces, let $\phi: C_{p}(X) \rightarrow C_{p}(Y)$ (resp. $\phi: C_{p}^{*}(X) \rightarrow$ $C_{p}^{*}(Y)$ ) be a continuous linear function and let $y \in Y$ be fixed. Notice that the function $\psi_{y}: C_{p}(X) \rightarrow \mathbb{R}$ (resp. $\left.\psi_{y}: C_{p}^{*}(X) \rightarrow \mathbb{R}\right)$ defined by $\psi_{y}(f)=\phi(f)(y)$

Received by the editors October 5, 1991.

1991 Mathematics Subject Classification. Primary 54C35, 57N17.

Key words and phrases. Function spaces, completely metrizable spaces.

The third author is pleased to thank the Department of Mathematics of the Vrije Universiteit (Amsterdam) for generous hospitality and support during his visit in 1990. 
is continuous and linear. So $\psi_{y} \in L(X)$ (resp. $\left.\psi_{y} \in L^{*}(X)\right)$, the dual of $C_{p}(X)$ (resp. $\left.C_{p}^{*}(X)\right)$. Since the evaluation mappings $\xi_{x}: C_{p}(X) \rightarrow \mathbb{R}(x \in X)$ defined by $\xi_{x}(f)=f(x)$ for $f \in C_{p}(X)$ (resp. $\left.f \in C_{p}^{*}(X)\right)$, form a Hamel basis for $L(X)$ (resp. $\left.L^{*}(X)\right)$, (cf. [14]), there are for $\psi_{y} \neq 0, x_{1}, \ldots, x_{n} \in X$ and $\lambda_{1}, \ldots, \lambda_{n} \in \mathbb{R} \backslash\{0\}$ such that $\psi_{y}=\sum_{i=1}^{n} \lambda_{i} \xi_{x_{i}}$. We define the support of $y$ in $X$ to be the finite set $\operatorname{supp}(y)=\left\{x_{1}, \ldots, x_{n}\right\} \subset X$. If $\psi_{y}=0$, the support of $y$ is defined to be the empty set (notice that whenever $\phi$ is onto, $\psi_{y} \neq 0$ for every $y \in Y)$. For $A \subset Y$, we abbreviate $\bigcup\{\operatorname{supp}(y): y \in A\}$ by $\operatorname{supp} A$. The notion of support here is a special case of a more general definition given by Arhangel'skii in [1]. The proof of the following lemma is a triviality and is only included for reference.

Lemma 2.1. Let $X$ and $Y$ be metric spaces, and let $\phi: C_{p}(X) \rightarrow C_{p}(Y)$ (resp. $\left.\phi: C_{p}^{*}(X) \rightarrow C_{p}^{*}(Y)\right)$, be a continuous linear function. Then for $y \in Y$,

1. for every $z \in \operatorname{supp}(y)$, there is $\lambda_{z} \in \mathbb{R}$ such that

$$
\phi(f)(y)=\sum_{z \in \operatorname{supp}(y)} \lambda_{z} f(z),
$$

for every $f \in C_{p}(X)$ (resp. $f \in C_{p}^{*}(X)$ ), and

2. if $f, g \in C_{p}(X)$ (resp. $\left.f, g \in C_{p}^{*}(X)\right)$, coincide on $\operatorname{supp}(y)$, then $\phi(f)(y)=\phi(g)(y)$.

If moreover $\phi$ is a linear homeomorphism, then supp $Y=X$.

Proposition 2.2 [1]. Let $X$ and $Y$ be metric spaces and let $\phi: C_{p}(X) \rightarrow C_{p}(Y)$ be a continuous linear function. If $A$ is a compact subset of $Y$, then $\overline{\operatorname{supp} A}$ is a compact subset of $X$.

Let $X$ and $Y$ be metric spaces and let $F: X \rightarrow \mathscr{P}(Y) \backslash\{\varnothing\}$ be a set-valued function such that for every $x \in X, F(x)$ is closed in $Y$. Then $F$ is said to be Lower Semi Continuous (abbreviated LSC) whenever for every open $U \subset$ $Y$ the set $\{x \in X: F(x) \cap U \neq \varnothing\}$ is open in $X$. If $\phi: C_{p}(X) \rightarrow C_{p}(Y)$ (resp. $\phi: C_{p}^{*}(X) \rightarrow C_{p}^{*}(Y)$ ) is a continuous linear function we can consider supp: $Y \rightarrow \mathscr{P}(X)$ as a set-valued function. We have

Proposition 2.3. Let $X$ and $Y$ be metric spaces and let $\phi: C_{p}(X) \rightarrow C_{p}(Y)$ (resp. $\phi: C_{p}^{*}(X) \rightarrow C_{p}^{*}(Y)$ ) be a continuous linear surjection. Then supp: $Y \rightarrow$ $\mathscr{P}(X) \backslash\{\phi\}$ is LSC.

Proof. Since $\phi$ is onto, $\operatorname{supp}(y) \neq \varnothing$ for every $y \in Y$. Of course, being a finite set, $\operatorname{supp}(y)$ is closed. Let $U$ be an open subset of $X$. Put $O=\{y \in$ $Y: \operatorname{supp}(y) \cap U \neq \varnothing\}$, and let $y \in O$. Then there is $x \in \operatorname{supp}(y) \cap U$. Let $f \in C_{p}^{*}(X)$ be such that $f((X \backslash U) \cup(\operatorname{supp}(y) \backslash\{x\}))=0$ and $f(x)=1$. Then by Lemma $2.1, \phi(f)(y)=\lambda_{x} \neq 0$. Let $W=\{z \in Y: \phi(f)(z) \neq 0\}$. Then $W$ is an open neighborhood of $y$. We claim that $W \subset O$. Suppose there is $z \in W \backslash O$, i.e., $\phi(f)(z) \neq 0$ and $\operatorname{supp}(z) \cap U=\varnothing$. But then $f(\operatorname{supp}(z))=0$, hence by Lemma $2.1 \phi(f)(z)=0$, which gives a contradiction, so $W \subset O$.

As usual for a cover $\mathscr{U}$ of a space $X$, we denote the cover $\{\operatorname{St}(U, \mathscr{U}): U \in$ $\mathscr{U}\}$ by $\operatorname{St}(\mathscr{U})$ and the cover $\operatorname{St}(\operatorname{St}(\mathscr{U}))$ by $\operatorname{St}^{2}(\mathscr{U})$. The next lemma will be very useful in the proof of Theorem 4.2. 
Lemma 2.4. Let $X$ be a metric space which is not completely metrizable. Let $\left\{\mathscr{R}_{n}: n \in \mathbb{N}\right\}$ and $\left\{\mathscr{S}_{n}: n \in \mathbb{N}\right\}$ be two collections of open covers of $X$ such that for each $n \in \mathbb{N}$,

(1) $\mathscr{R}_{n}$ and $\mathscr{S}_{n}$ are locally finite,

(2) $\operatorname{diam} \mathscr{R}_{n}<1 / n$,

(3) $\operatorname{St}^{2}\left(\mathscr{R}_{n+1}\right) \prec \mathscr{S}_{n} \prec \mathscr{R}_{n}$,

(4) each element of $\mathscr{S}_{n+1}$ intersects only finitely many elements of $\mathscr{S}_{n}$.

Then for each $n \in \mathbb{N}$ we can find $S_{n} \in \mathscr{S}_{n}$ such that $\operatorname{St}\left(\overline{S_{n+1}}, \mathscr{R}_{n+1}\right) \subset S_{n}$ and $\bigcap_{n=1}^{\infty} S_{n}=\varnothing$.

Proof. Let $\tilde{X}$ be the completion of $X$. For each $n \in \mathbb{N}$ and for each $S \in \mathscr{S}_{n}$ we can find $V_{S}$ open in $\tilde{X}$ such that

(5) $\operatorname{diam} V_{S}<3 / n$,

(6) $V_{S} \cap X=S$,

(7) if $S \in \mathscr{S}_{n}, T \in \mathscr{S}_{n+1}$ and $T \subset S$, then $V_{T} \subset V_{S}$.

Indeed, suppose for each $m \leq n$ and $S \in \mathscr{S}_{m}$ we found $V_{S}$. Now let $S \in$ $\mathscr{S}_{n+1}$. By (4), there are only finitely many elements of $\mathscr{S}_{n}$ which contain $S$. Find $O$ open in $X$ such that $\operatorname{diam} O<3 /(n+1)$ and $O \cap X=S$. Let $V_{S}=O \cap \bigcap\left\{V_{T}: T \in \mathscr{S}_{n}\right.$ with $\left.S \subset T\right\}$. Then this $V_{S}$ obviously satisfies conditions (5), (6) and (7). This completes the inductive construction.

For each $n \in \mathbb{N}$ let $V_{n}=\bigcup\left\{V_{S}: S \in \mathscr{S}_{n}\right\}$. Then $V_{n}$ is open in $\tilde{X}$. So $V=\bigcap_{n=1}^{\infty} V_{n}$ is a $G_{\delta}$-subset of $\widetilde{X}$, hence completely metrizable. Since $X \subset V$, this implies that there exists $x \in V \backslash X$. For each $n \in \mathbb{N}$, let $\mathscr{U}_{n}=\left\{V_{S}: S \in \mathscr{S}_{n}\right.$ with $\left.x \in V_{S}\right\}$ and $\mathscr{V}_{n}=\left\{S: V_{S} \in \mathscr{U}_{n}\right\}$. Then $\mathscr{U}_{n}$ is a finite family. Indeed, let $T \in \mathscr{S}_{n+1}$ be such that $x \in V_{T}$. Then for each $S \in \mathscr{V}_{n}, x \in V_{S} \cap V_{T}$. Since $x \in \bar{X}, V_{S} \cap V_{T} \cap X \neq \varnothing$ which gives $S \cap T \neq \varnothing$. Since $T$ intersects only finitely many elements of $\mathscr{S}_{n}$, we are done.

Now for $S \in \mathscr{V}_{n}$ and $T \in \mathscr{V}_{n+1}$ we define

$$
T \preceq S \Leftrightarrow_{\text {def }} \operatorname{St}\left(\bar{T}, \mathscr{R}_{n+1}\right) \subset S .
$$

We claim that this ordering gives us chains of arbitrary length. For if $T \in \mathscr{V}_{n+1}$, there exists by (3) $S \in \mathscr{S}_{n}$ such that $\operatorname{St}\left(\bar{T}, \mathscr{R}_{n+1}\right) \subset S$. Since $T \subset S$ we have by (3), $V_{T} \subset V_{S}$, hence $S \in \mathscr{V}_{n}$ and we can walk all the way up to $\mathscr{V}_{1}$. Now by Königs lemma [12, Lemma II.5.7], we have an infinite chain with respect to this ordering, say $\left\{S_{n}: n \in \mathbb{N}\right\}$ with $S_{n} \in \mathscr{S}_{n}$. Since diam $V_{S_{n}} \rightarrow 0$, $\{x\}=\bigcap_{n=1}^{\infty} V_{S_{n}}$, and hence $\bigcap_{n=1}^{\infty} S_{n}=\varnothing$.

A weaker version of this lemma will be used in the proof of Theorem 3.3 (its proof is similar).

Lemma 2.5. Let $X$ be a metric space which is not completely metrizable. Let $\left\{\mathscr{U}_{n}: n \in \mathbb{N}\right\}$ be a collection of locally finite open covers of $X$ such that for each $n \in \mathbb{N}$, diam $\mathscr{U}_{n}<\frac{1}{n}$ and $\operatorname{St}\left(\mathscr{U}_{n+1}\right) \prec \mathscr{U}_{n}$. Then for each $n \in \mathbb{N}$, there is $U_{n} \in \mathscr{U}_{n}$ such that $\overline{U_{n+1}} \subset U_{n}$, and moreover $\bigcap_{n=1}^{\infty} U_{n}=\varnothing$.

\section{THE UNBOUNDED CASE}

Lemma 3.1. Let $X$ and $Y$ be metric spaces, and let $\phi: C_{p}(X) \rightarrow C_{p}(Y)$ be a continuous linear surjection. Then for each compact $K \subset X$, the set $L=\{y \in$ $Y: \operatorname{supp}(y) \subset K\}$ is a compact subset of $Y$. 
Proof. By Proposition 2.3, supp is LSC, hence $L$ is closed. If $L$ is not compact, $L$ contains a closed discrete subset $\left\{y_{n}: n \in \mathbb{N}\right\}$. For each $n \in \mathbb{N}$, let $t_{n}=$ $n \cdot \sum_{z \in \operatorname{supp}\left(y_{n}\right)}\left|\lambda_{z}\right|$. Then $t_{n}>0$. Let $g \in C_{p}(Y)$ be such that $g\left(y_{n}\right)=t_{n}$. Since $\phi$ is a surjection, there is $f \in C_{p}(X)$ such that $\phi(f)=g$. Since $K$ is compact, there is $c \in \mathbb{R}$ such that $f(K) \subset[-c, c]$. Let $n \in \mathbb{N}$ be such that $n>c$. Then

$$
\begin{aligned}
\left|\phi(f)\left(y_{n}\right)\right| & =\left|\sum_{z \in \operatorname{supp}\left(y_{n}\right)} \lambda_{z} \cdot f(z)\right| \leq \sum_{z \in \operatorname{supp}\left(y_{n}\right)}\left|\lambda_{z}\right| \cdot|f(z)| \\
& \leq c \cdot \sum_{z \in \operatorname{supp}\left(y_{n}\right)}\left|\lambda_{z}\right|<t_{n},
\end{aligned}
$$

so we arrive at a contradiction.

Let $X$ and $Y$ be metric spaces and let $\phi: C_{p}(X) \rightarrow C_{p}(Y)$ be a continuous linear surjection. For $U \subset X$, let $T_{U}=\{y \in Y: \operatorname{supp}(y) \cap U \neq \varnothing\}$. For a family $\mathscr{U}$ of subsets of $X$, let $T_{\mathscr{U}}=\left\{T_{U}: U \in \mathscr{U}\right\}$.

Lemma 3.2. If $\mathscr{U}$ is a locally finite open cover of $X$, then $T_{\mathscr{U}}$ is a locally finite open cover of $Y$.

Proof. Since $\phi$ is onto we have for each $y \in Y, \operatorname{supp}(y) \neq \varnothing$. So $T_{\mathscr{U}}$ covers $Y$. Furthermore by Lemma 2.3, supp is LSC, so for $U \in \mathscr{U}, T_{U}$ is open in $Y$. If $T_{\mathscr{U}}$ is not locally finite there are $y \in Y$, a sequence $y_{n} \rightarrow y$ $(n \rightarrow \infty)$, and distinct $U_{n}$ 's in $\mathscr{U}$ such that $y_{n} \in T_{U_{n}}$. Let $x_{n} \in \operatorname{supp}\left(y_{n}\right) \cap U_{n}$. Since $\overline{\left\{y_{n}: n \in \mathbb{N}\right\}}$ is compact, by Proposition 2.2, $\overline{\operatorname{supp}\left\{y_{n}: n \in \mathbb{N}\right\}}$ is compact. Hence $\overline{\left\{x_{n}: n \in \mathbb{N}\right\}}$ is compact. Since $\mathscr{U}$ is locally finite, $\overline{\left\{x_{n}: n \in \mathbb{N}\right\}}$ intersects only finitely many elements of $\mathscr{U}$. Contradiction.

Theorem 3.3. Let $X$ and $Y$ be metric spaces and let $\phi: C_{p}(X) \rightarrow C_{p}(Y)$ be a continuous linear surjection. If $X$ is completely metrizable, then $Y$ is completely metrizable.

Proof. Suppose $X$ is completely metrizable and $Y$ is not completely metrizable. Provide $X$ with a complete metric. Since $X$ and $Y$ are metric spaces, by Lemma 3.2 there are locally finite open covers $\mathscr{U}_{n}$ of $X$ and $\mathscr{V}_{n}$ of $Y$, $(n \in \mathbb{N})$ such that

(1) $\operatorname{diam} \mathscr{U}_{n}<\frac{1}{n}, \operatorname{diam} \mathscr{V}_{n}<\frac{1}{n}, \mathscr{U}_{n+1} \prec \mathscr{U}_{n}, \operatorname{St}\left(\mathscr{V}_{n+1}\right) \prec \mathscr{V}_{n}$, and

(2) each $V \in \mathscr{V}_{n}$ intersects only finitely many elements of $T_{\mathscr{U}_{n}}$.

By Lemma 2.5, for each $n \in \mathbb{N}$, there is $V_{n} \in \mathscr{V}_{n}$ such that $\bigcap_{n=1}^{\infty} V_{n}=\varnothing$ and for each $n \in \mathbb{N}, \bar{V}_{n+1} \subset V_{n}$. By (2), for each $n \in \mathbb{N}$, there is a finite subset $\left\{U_{1}^{n}, \ldots, U_{m_{n}}^{n}\right\}$ of $\mathscr{U}_{n}$ such that

(3) for $U \in \mathscr{U}_{n}, V_{n} \cap T_{U} \neq \varnothing$ if and only if $U \in\left\{U_{1}^{n}, \ldots, U_{m_{n}}^{n}\right\}$.

We claim that for each $n \in \mathbb{N}$,

(4) $\bigcup_{j=1}^{m_{n+1}} U_{j}^{n+1} \subset \bigcup_{j=1}^{m_{n}} U_{j}^{n}$.

Indeed, since $\mathscr{U}_{n+1}$ refines $\mathscr{U}_{n}$, there are for each $j \leq m_{n+1}, U_{j} \in \mathscr{U}_{n}$ such that $U_{j}^{n+1} \subset U_{j}$. Since $V_{n+1} \subset V_{n}$, and $V_{n+1} \cap T_{U_{j}^{n+1}} \neq \varnothing$, we have $V_{n} \cap T_{U_{j}} \neq \varnothing$. So by (3), $U_{j} \in\left\{U_{1}^{n}, \ldots, U_{m_{n}}^{n}\right\}$. This gives $\bigcup_{j=1}^{m_{n+1}} U_{j}^{n+1} \subset \bigcup_{j=1}^{m_{n}} U_{j}^{n}$. This proves (4). Notice that by (3), for every $n \in \mathbb{N}$, supp $V_{n} \subset \bigcup_{j=1}^{m_{n}} U_{j}^{n}$. For each $n \in \mathbb{N}$, let $y_{n} \in V_{n}$. Then 
(5) $\operatorname{supp}\left(y_{n}\right) \subset \bigcup_{j=1}^{m_{n}} U_{j}^{n}$.

Let $K=\overline{\bigcup_{n=1}^{\infty} \operatorname{supp}\left(y_{n}\right)}$. Since $K$ is a closed subset of $X, K$ is complete.

Claim. $K$ is compact.

Since $K$ is complete, it remains to prove that $K$ is totally bounded. To this end it suffices to prove that $\bigcup_{n=1}^{\infty} \operatorname{supp}\left(y_{n}\right)$ is totally bounded. Let $\varepsilon>0$ and let $j \in \mathbb{N}$ be such that $\frac{1}{j}<\frac{\varepsilon}{2}$. For $k \leq m_{j}$, let $z_{k} \in U_{k}^{j}$. Since $\operatorname{diam} U_{k}^{j}<\frac{1}{j}$, $U_{k}^{j} \subset B\left(z_{k}, \varepsilon\right)$. Then by (4) and (5), $\bigcup_{n=j}^{\infty} \operatorname{supp}\left(y_{n}\right) \subset \bigcup_{k=1}^{m_{j}} B\left(z_{k}, \varepsilon\right)$. Since $\bigcup_{n=1}^{j} \operatorname{supp}\left(y_{n}\right)$ is finite, we are done.

By Lemma 3.1, $L=\{y \in Y: \operatorname{supp}(y) \subset K\}$ is a compact subset of $Y$. Since $\bigcap_{n=1}^{\infty} \bar{V}_{n}=\varnothing,\left(y_{n}\right)_{n \in \mathbb{N}} \subset L$ is a sequence without convergent subsequence. Contradiction. This proves the theorem.

Corollary 3.4. Complete metrizability is preserved by $l_{p}$-equivalence in the class of metric spaces.

Let $\mathbb{Q}$ denote the space of rationals. Let $T=\mathbb{N}^{2} \cup\{\infty\}$, where each point of $\mathbb{N}^{2}$ is isolated and $\{(\{n, n+1, \ldots\} \times \mathbb{N}) \cup\{\infty\}\}_{n \in \mathbb{N}}$ is a local base at $\infty$. In [7] it was shown that $\mathbb{Q}$ and $T$ are not $l_{p}$-equivalent. This result now also follows from Corollary 3.4 .

In [18], Uspenskii proved for $l_{p}$-equivalent spaces $X$ and $Y$, with $X$ metric, that $Y$ is a $\sigma$-metrizable paracompact space, where $\sigma$-metrizable means a countable union of closed metrizable subspaces. In view of Theorem 3.3, one could conjecture that if $X$ is moreover completely metrizable, then $Y$ is also Čech-complete. This is however not the case. Take for $X$ the ordinal space $\omega^{2}$ and for $Y$ the space obtained from $X$ by identifying all accumulation points of $X$ to one point. The spaces $X$ and $Y$ are $l_{p}$-equivalent (see for example [5]). The space $X$ is countable metric and locally compact while $Y$ is paracompact and $\sigma$-metrizable but not Čech-complete.

In the next section we prove the same result for spaces $C_{p}^{*}(X)$ : if $X$ and $Y$ are metric spaces and $\phi: C_{p}^{*}(X) \rightarrow C_{p}^{*}(Y)$ is a continuous linear surjection, then $Y$ is completely metrizable provided $X$ is. In this case a proof like Theorem 3.3 seems not to be working. We give an example which shows that we cannot make use of a result like Lemma 3.2. Let $\left(x_{n}\right)_{n \in \mathbb{N}}$ be a convergent sequence, say $x_{n} \rightarrow x \quad(n \rightarrow \infty)$. Let $Y=\left\{x_{n}: n \in \mathbb{N}\right\} \cup\{x\}$, and let $X=Y \oplus \mathbb{N}$ the topological sum of $Y$ and $\mathbb{N}$. Define $\phi: C_{p}^{*}(X) \rightarrow C_{p}^{*}(Y)$ by

$$
\phi(f)(z)= \begin{cases}f\left(x_{n}\right)+\frac{1}{n} \cdot f(n) & \text { if } z=x_{n} \\ f(x) & \text { if } z=x\end{cases}
$$

Since $f$ is bounded, we have $\phi(f) \in C_{p}^{*}(Y)$. It is easily seen that $\phi$ is a linear continuous surjection. Let $\mathscr{U}=\{Y\} \cup\{\{n\}: n \in \mathbb{N}\}$. Then $\mathscr{U}$ is a locally finite open cover of $X$. Since for every $n \in \mathbb{N}, \operatorname{supp}\left(x_{n}\right)=\left\{n, x_{n}\right\}, T_{\mathscr{U}}$ is not locally finite.

Acknowledgments. We would like to express our gratitude to Roman Pol who originally proved that the space of irrationals $\mathbb{P}$ and the topological sum of $\mathbb{P}$ and the space of rationals $\mathbb{Q}$ are not $l_{p}$-equivalent. 


\section{THE BOUNDED CASE}

Lemma 4.1. Let $X$ and $Y$ be metric spaces and let $\phi: C_{p}^{*}(X) \rightarrow C_{p}^{*}(Y)$ be a continuous linear function and let $\mathscr{P}$ be a nonempty locally finite family of open subsets of $X$. Then for each $y \in Y$ and for each $\varepsilon>0$, there exists a neighborhood $U$ of $y$ and a nonempty finite subset $\mathscr{F}=\mathscr{F}(\varepsilon, U, \mathscr{P})$ of $\mathscr{P}$ such that for each $z \in U$,

$$
\sum\left\{\left|\lambda_{a}\right|: a \in(\operatorname{supp}(z) \backslash \bigcup \mathscr{F}) \cap \bigcup \mathscr{P}\right\}<\varepsilon .
$$

Proof. Suppose the lemma is false. Find $y \in Y$ and $\varepsilon_{0}>0$ contradicting the lemma. For each $x \in \bigcup \mathscr{P}$, let $\mathscr{P}_{x}=\{P \in \mathscr{P}: x \in P\}$. Obviously $\mathscr{P}_{x}$ is finite. For each $x \in \bigcup \mathscr{P}$, find a neighborhood $W_{x}$ of $x$ such that $W_{x}$ intersects only finitely many elements of $\mathscr{P}$ and such that $\bar{W}_{x} \subset \cap \mathscr{P}_{x}$. Since $\operatorname{supp}(y)$ is finite, there is a finite subset $\mathscr{F}_{1}$ of $\mathscr{P}$ such that $\operatorname{supp}(y) \cap \bigcup \mathscr{P} \subset \bigcup \mathscr{F}$. Let $W_{0}=\bigcup\left\{W_{x}: x \in \operatorname{supp}(y) \cap \bigcup \mathscr{P}\right\}$. By induction we construct a decreasing open base $\left\{U_{n}: n \in \mathbb{N}\right\}$ at $y$, for each $n \in \mathbb{N}$ we find points $z_{n} \in U_{n}$ and functions $f_{n} \in C_{p}^{*}(X)$ such that if for each $n \in \mathbb{N}$,

$$
\begin{aligned}
& M_{n}=\left(\operatorname{supp}\left(z_{n}\right) \cap \bigcup \mathscr{P}\right) \backslash \bigcup \mathscr{F}_{n}, \\
& W_{n}=\bigcup\left\{W_{x}: x \in M_{n}\right\} \backslash\left(\bigcup\left\{\bar{W}_{i}: i \leq n-1\right\} \cup \bigcup\left\{\operatorname{supp}\left(z_{i}\right) \cap \bigcup \mathscr{F}_{i}: i \leq n\right\}\right), \\
& \mathscr{T}_{n}=\bigcup\left\{\mathscr{P}_{x}: x \in M_{n}\right\}, \\
& \mathscr{F}_{n+1}=\mathscr{F}_{n} \cup \mathscr{T}_{n}, \text { and } \\
& \delta_{n}=\sum_{a \in M_{n}}\left|\lambda_{a}\right|,
\end{aligned}
$$

then the following properties are fulfilled

(1) $\delta_{n} \geq \varepsilon_{0}$

(2) $f_{n}\left(X \backslash W_{n}\right)=\{0\}, f_{n}(a)=\operatorname{sign}\left(\lambda_{a}\right) \cdot 1 / \delta_{n}$ for $a \in M_{n},\left\|f_{n}\right\| \leq 1 / \varepsilon_{0}$, and

(3) $\phi\left(f_{n}\right)\left(U_{n+1}\right) \subset\left(-1 / 2^{n+1}, 1 / 2^{n+1}\right)$.

(It will be convenient for our inductive construction to observe that each $\mathscr{F}_{n}$ is a finite subcollection of $\mathscr{P}$, each $M_{n}$ is finite and moreover a subset of $W_{n}$ since for each $i \leq n-1, \bar{W}_{i} \subset \bigcup \mathscr{T}_{i} \subset \bigcup \mathscr{F}_{n}$.)

For $n=1$, let $U_{1}$ be an arbitrary neighborhood of $y$. Since $\varepsilon_{0}$ contradicts the lemma we can find $z_{1} \in U_{1}$ be such that $\delta_{1} \geq \varepsilon_{0}$. Define $g_{1}:\left(X \backslash W_{1}\right) \cup$ $M_{1} \rightarrow\left[0,1 / \varepsilon_{0}\right]$ by $g_{1}(x)=0$ for $x \in X \backslash W_{1}$ and $g_{1}(x)=\operatorname{sign}\left(\lambda_{x}\right) \cdot 1 / \delta_{1}$ for $x \in M_{1}$. Since $M_{1} \subset W_{1}$ is finite, $g_{1}$ is continuous. Extend $g_{1}$ to a continuous $f_{1}: X \rightarrow\left[0,1 / \varepsilon_{0}\right]$. Then $f_{1}$ satisfies condition (2). Since condition (3) is empty for $n=1$, the initial step of the construction is completed.

Suppose we found $f_{1}, \ldots, f_{n}, z_{1}, \ldots, z_{n}$, and $U_{1}, \ldots, U_{n}$. Since $\operatorname{supp}(y) \cap$ $W_{n}=\varnothing, f_{n}(\operatorname{supp}(y))=\{0\}$, hence by Lemma $2.1 \phi\left(f_{n}\right)(y)=0$. Thus by continuity of $f$ we can find a neighborhood $U_{n+1} \subset U_{n}$ of $y$ such that

$$
\phi\left(f_{n}\right)\left(U_{n+1}\right) \subset\left(\frac{-1}{2^{n+1}}, \frac{1}{2^{n+1}}\right) .
$$

Since $\varepsilon_{0}$ contradicts the lemma we can find $z_{n+1} \in U_{n+1}$ such that $\delta_{n+1} \geq \varepsilon_{0}$. The function $f_{n+1}$ can be constructed in the same way as $f_{1}$. This completes the inductive construction.

Claim. $\left\{W_{n}: n \in \mathbb{N}\right\}$ is a discrete family in $X$.

Since the family $\left\{W_{n}: n \in \mathbb{N}\right\}$ is pairwise disjoint it suffices to show that the family is locally finite. Let $x \in M_{n}$ and $y \in M_{m}$, with $m>n$. Since $\mathscr{P}_{x} \subset \mathscr{T}_{n} \subset \mathscr{F}_{n+1}$ and $y \notin \bigcup \mathscr{F}_{n+1}, \mathscr{P}_{x} \cap \mathscr{P}_{y}=\varnothing$. Now let $z \in X$ and $U$ a neighborhood of $z$ that intersects only finitely many elements of $\mathscr{P}$. The 
above observation gives that there exists $m \in \mathbb{N}$ such that $\bigcup\left\{\mathscr{P}_{x}: x \in M_{n}\right.$ for some $n>m\} \cap U=\varnothing$. Let $n>m$ and suppose $U \cap W_{n} \neq \varnothing$. Then there is $x \in M_{n}$ such that $U \cap W_{x} \neq \varnothing$. Since $W_{x} \subset \cap \mathscr{P}_{x}$, we arrive at a contradiction, hence $U$ can only intersect finitely many elements of our family $\left\{W_{n}: n \in \mathbb{N}\right\}$.

From (1) we get $M_{n} \neq \varnothing$. Moreover it easily follows that for $n \in \mathbb{N}$, $\operatorname{supp}\left(z_{n}\right) \cap W_{n}=M_{n}$ and for $m>n, \operatorname{supp}\left(z_{n}\right) \cap W_{m}=\varnothing$.

Now let $f=\sum_{n=1}^{\infty} f_{n}$. By the claim we have $f \in C_{p}^{*}(X)$, so $\phi(f) \in C_{p}^{*}(Y)$. Since $f(\operatorname{supp}(y))=0, \phi(f)(y)=0$, hence $\phi(f)\left(z_{n}\right) \rightarrow 0(n \rightarrow \infty)$. However

$$
\phi\left(f_{n}\right)\left(z_{n}\right)=\sum_{a \in \operatorname{supp}\left(z_{n}\right)} \lambda_{a} f_{n}(a)=\sum_{a \in M_{n}} \lambda_{a} f_{n}(a)=\frac{1}{\delta_{n}} \cdot \delta_{n}=1
$$

and

$$
\phi\left(f_{m}\right)\left(z_{n}\right)=\sum_{a \in \operatorname{supp}\left(z_{n}\right)} \lambda_{a} f_{m}(a)=0 \text { since } \operatorname{supp}\left(z_{n}\right) \cap W_{m}=\varnothing \text { for } m>n .
$$

So finally we get

$$
\begin{aligned}
\left|\phi(f)\left(z_{n}\right)\right| & =\left|\sum_{m=1}^{\infty} \phi\left(f_{m}\right)\left(z_{n}\right)\right|=\left|\sum_{m=1}^{n-1} \phi\left(f_{m}\right)\left(z_{n}\right)+\phi\left(f_{n}\right)\left(z_{n}\right)\right| \\
& \geq 1-\sum_{m=1}^{n-1}\left|\phi\left(f_{m}\right)\left(z_{n}\right)\right| \geq 1-\sum_{m=1}^{n-1} \frac{1}{2^{m+1}} \geq \frac{1}{2} .
\end{aligned}
$$

We arrive at a contradiction and hence the lemma is true (note that $\mathscr{F}$ can be assumed to be nonempty since we can always add one extra member of $\mathscr{P}$ to $\mathscr{F})$.

In the proof of the forthcoming theorem we make use of two well-known theorems from the theory of Banach spaces.The first one is the Closed Graph Theorem, which states that for Banach spaces $E$ and $F$ and a linear function $\phi: E \rightarrow F$ such that the set $\{(x, \phi(x): x \in E\}$ is closed in $E \times F$, we have $\phi$ is continuous [11, Theorem X.4.1]. The second one is the Open Mapping Theorem, which states that a continuous linear function of a Banach space $E$ onto a Banach space $F$ is open [13, Theorem 1.4.10]. We denote $C^{*}(X)$ with the topology of uniform convergence by $C_{u}^{*}(X)$. It is well known that $C_{u}^{*}(X)$ is a Banach space.

If $C=\left[U_{1}, \ldots, U_{n}, U\right]$ is a sequence of sets, then we abbreviate $\left[U_{1}, \ldots, U_{n}\right]$ by $C \downarrow U$. We use the symbol " $\wedge$ " to denote the intersection of families of sets.

Theorem 4.2. Let $X$ and $Y$ be metric spaces and let $\phi: C_{p}^{*}(X) \rightarrow C_{p}^{*}(Y)$ be a continuous linear surjection. If $X$ is completely metrizable, then $Y$ is completely metrizable.

Proof. Suppose $X$ is completely metrizable and $Y$ is not completely metrizable. Provide $X$ with a complete metric $d$. By the Closed Graph Theorem, $\phi$ considered as a map from $C_{u}^{*}(X)$ onto $C_{u}^{*}(Y)$ is continuous. This, together with the Open Mapping Theorem gives us $k \in \mathbb{N}$ such that for all $f \in C^{*}(X)$ with $\|f\|=1,\|\phi(f)\| \leq k$, and for all $g \in C^{*}(Y)$ with $\|g\|=1$, there is $f \in \phi^{-1}(g)$ with $\|f\| \leq k$. 
Claim 1. For each $y \in Y, \sum_{a \in \operatorname{supp}(y)}\left|\lambda_{a}\right| \leq k$.

Let $f \in C_{p}^{*}(X)$ be such that $\|f\|=1$ and for all $a \in \operatorname{supp}(y), f(a)=$ $\operatorname{sign}\left(\lambda_{a}\right)$. Then $\|\phi(f)\| \leq k$, hence $\phi(f)(y)=\sum_{a \in \operatorname{supp}(y)} \lambda_{a} f(a)=\sum_{a \in \operatorname{supp}(y)}\left|\lambda_{a}\right|$ $\leq k$.

Let $\left(\mathscr{P}_{n}\right)_{n \in \omega}$ be a sequence of open covers of $X$ such that for each $n \in \omega$,

(1) $\mathscr{P}_{n+1} \prec \mathscr{P}_{n}$,

(2) each $\mathscr{P}_{n}$ is locally finite,

(3) $\operatorname{diam} \mathscr{P}_{n}<2^{-n}$.

For each $n \in \mathbb{N}$, let $\varepsilon_{n}=1 /\left(k \cdot 2^{n+3}\right)$. By induction we construct sequences $\left(\mathscr{R}_{n}\right)_{n \in \omega}$ and $\left(\mathscr{S}_{n}\right)_{n \in \omega}$ of open covers of $Y$, sets of sequences given by

$$
\begin{aligned}
& \mathscr{C}_{0}=\left\{[U]: U \in \mathscr{R}_{0}\right\}, \text { and for } n>0, \\
& \mathscr{C}_{n}=\left\{\left[U_{0}, \ldots, U_{n}\right]:\left[U_{0}, \ldots, U_{n-1}\right] \in \mathscr{C}_{n-1}, U_{n} \in \mathscr{R}_{n} \text { and } U_{n} \subset U_{n-1}\right\},
\end{aligned}
$$

and for each $C \in \mathscr{C}_{n}$ a nonempty finite set $\mathscr{Z}(C, n)$ of open subsets of $X$ such that

(4) $\mathscr{R}_{n}$ and $\mathscr{S}_{n}$ are locally finite,

(5) $\operatorname{diam} \mathscr{R}_{n}<1 /(n+1)$,

(6) $\operatorname{St}^{2}\left(\mathscr{R}_{n+1}\right) \prec \mathscr{S}_{n} \prec \mathscr{R}_{n}$,

(7) each element of $\mathscr{S}_{n+1}$ intersects only finitely many elements of $\mathscr{S}_{n}$,

(8) if $C \in \mathscr{C}_{n}$, then each $V \in \mathscr{Z}(C, n)$ is contained in a member of $\mathscr{P}_{n}$,

(9) if $C=\left[U_{0}, \ldots, U_{n}, U\right] \in \mathscr{C}_{n+1}$, then

$$
\begin{gathered}
\bigcup \mathscr{Z}(C, n+1) \subset \bigcup \mathscr{Z}(C \downarrow U, n) \text { and for } z \in U, \\
\sum\left\{\left|\lambda_{x}\right|: x \in(\operatorname{supp}(z) \backslash \bigcup \mathscr{Z}(C, n+1)) \cap \bigcup \mathscr{Z}(C \downarrow U, n)\right\}<\varepsilon_{n+1} .
\end{gathered}
$$

(10) for each $U \in \mathscr{R}_{0}$ and $z \in U, \sum\left\{\left|\lambda_{x}\right|: x \in \operatorname{supp}(z) \backslash \bigcup \mathscr{Z}([U], 0)\right\}<$ $\varepsilon_{0}$.

By Lemma 4.1 there exists a locally finite open cover $\mathscr{R}_{0}$ of $Y$ with $\operatorname{diam} \mathscr{R}_{0}<$ 1 and for each $U \in \mathscr{R}_{0}$ a nonempty finite subset $\mathscr{F}=\mathscr{F}\left(\varepsilon_{0}, U, \mathscr{P}_{0}\right)$ of $\mathscr{P}_{0}$ such that for each $z \in U$,

$$
\sum\left\{\left|\lambda_{a}\right|: a \in \operatorname{supp}(z) \backslash \bigcup \mathscr{F}\right\}<\varepsilon_{0} .
$$

Put $\mathscr{Z}([U], 0)=\mathscr{F}\left(\varepsilon_{0}, U, \mathscr{P}_{0}\right)$. Let $\mathscr{S}_{0}$ be a locally finite open cover of $X$ refining $\mathscr{R}_{0}$ and such that each element of $\mathscr{S}_{0}$ intersects only finitely many elements of $\mathscr{R}_{0}$.

Suppose for $n \in \omega, \mathscr{R}_{0}, \ldots, \mathscr{R}_{n}$ and $\mathscr{S}_{0}, \ldots, \mathscr{S}_{n}$ satisfy our requirements. Let $\mathscr{S}$ be a locally finite open cover of $Y$ such that $\operatorname{St}^{2}(\mathscr{S})$ refines $\mathscr{S}_{n}$ and each element of $\mathscr{S}$ intersects only finitely many elements of $\mathscr{R}_{n}$. For $C \in \mathscr{C}_{n}$, put $\mathscr{T}_{C}=\mathscr{P}_{n+1} \wedge \mathscr{Z}(C, n)$. Note that $\mathscr{T}_{C}$ is a locally finite open cover of $\bigcup \mathscr{Z}(C, n)$. Let $S \in \mathscr{S}$ and put $\mathscr{C}(S)=\left\{\left[U_{0}, \ldots, U_{n}\right] \in \mathscr{C}_{n}: S \cap U_{n} \neq \varnothing\right\}$. Then $\mathscr{C}(S)$ is finite and nonempty. Let $C \in \mathscr{C}(S)$. By Lemma 4.1, there exists an open cover $\mathscr{V}_{C}^{S}$ of $S$ and for each $V \in \mathscr{V}_{C}^{S}$ a nonempty finite subset $\mathscr{F}_{S}=\mathscr{F}_{S}\left(\varepsilon_{n+1}, V, \mathscr{T}_{C}\right)$ of $\mathscr{T}_{C}$ such that for each $z \in V$,

$$
\sum\left\{\left|\lambda_{z}\right|: x \in\left(\operatorname{supp}(z) \cap \bigcup \mathscr{T}_{C}\right) \backslash \bigcup \mathscr{F}_{S}\right\}<\varepsilon_{n+1} \text {. }
$$

Let $\mathscr{W}_{S}$ be a locally finite (in $X$ !) open cover of $S$ refining $\bigwedge\left\{\mathscr{V}_{C}^{S}: C \in \mathscr{C}(S)\right\}$ such that $\operatorname{diam} \mathscr{W}_{S}<1 /(n+1)$. Let $\mathscr{R}_{n+1}=\bigcup\left\{\mathscr{W}_{S}: S \in S\right\}$. Then $\mathscr{R}_{n+1}$ is 
a locally finite open cover of $Y$ such that $\operatorname{St}^{2}\left(\mathscr{R}_{n+1}\right)$ refines $\mathscr{S}_{n}$. For $C=$ $\left[U_{0}, \ldots, U_{n}, U\right] \in \mathscr{C}_{n+1}$, put $\mathscr{S}^{\prime}=\left\{S \in \mathscr{S}: S \cap U_{n} \neq \varnothing\right\}$ and define

$$
\mathscr{Z}(C, n+1)=\bigcup\left\{\mathscr{F}_{S}\left(\varepsilon_{n+1}, V, \mathscr{T}_{C \downarrow U}\right): S \in \mathscr{S}^{\prime} \text { with } U \subset V \in \mathscr{V}_{C \downarrow U}^{S}\right\} .
$$

Obviously $\bigcup \mathscr{Z}(C, n+1) \subset \mathscr{Z}(C \downarrow U, n)$ and $\mathscr{Z}(C, n)$ is nonempty and finite. Since $U \in \mathscr{R}_{n+1}$, there is $S \in \mathscr{S}$ with $U \in \mathscr{W}_{S}$. This gives $U \subset S$, hence $S \cap U_{n} \neq \varnothing$. So we have $C \downarrow U \in \mathscr{C}(S)$. Since $\mathscr{W}_{S}$ refines $\mathscr{V}_{C \downarrow U}^{S}$, there exists $V \in \mathscr{V}_{C \downarrow U}^{S}$ such that $U \subset V$. Then $\bigcup \mathscr{F}_{S}\left(\varepsilon, V, \mathscr{T}_{C \downarrow U}\right) \subset \mathscr{Z}(C, n+1)$. By $(*)$, we have $(9)$.

To finish the inductive construction, let $\mathscr{S}_{n+1}$ be a locally finite refinement of $\mathscr{R}_{n+1}$ such that each element of $\mathscr{S}_{n+1}$ intersects only finitely many elements of $\mathscr{S}_{n}$.

By Lemma 2.4, (4), (5), (6) and (7) there are $S_{n} \in \mathscr{S}_{n}$ such that for each $n \in \mathbb{N}$,

$\operatorname{St}\left(\overline{S_{n+1}}, \mathscr{R}_{n+1}\right) \subset S_{n}$ and $\bigcap_{n=1}^{\infty} S_{n}=\varnothing$.

For each $n \in \mathbb{N}$ find $U_{n} \in \mathscr{R}_{n}$ such that $S_{n} \subset U_{n}$. Then it easily follows that $U_{n+1} \subset S_{n}$. Let $C_{n}=\left[U_{0}, \ldots, U_{n}\right]$. For each $n \in \mathbb{N}$ and for each $V \in \mathscr{Z}\left(C_{n}, n\right)$ pick a point $x_{V} \in V$. Let

$$
K=\left(\bigcap_{n=1}^{\infty} \overline{\bigcup \mathscr{Z}\left(C_{n}, n\right)}\right) \cup\left(\overline{\bigcup_{n=1}^{\infty}\left\{x_{V}: V \in \mathscr{Z}\left(C_{n}, n\right)\right\}}\right) .
$$

Since $K$ is a closed subspace of $X, K$ is complete.

Claim 2. $K$ is compact.

Since $K$ is complete it suffices to prove that $K$ is totally bounded. To this end it suffices to prove that $\bigcup_{n=1}^{\infty}\left\{x_{V}: V \in \mathscr{Z}\left(C_{n}, n\right)\right\}$ is totally bounded and $\bigcap_{n=1}^{\infty} \overline{\cup \mathscr{Z}\left(C_{n}, n\right)}$ is totally bounded. Let $\varepsilon>0$ and let $j \in \mathbb{N}$ be such that $2^{-j}<\varepsilon / 2$. For $V \in \mathscr{Z}\left(C_{j}, j\right)$ we have by (8) diam $V<2^{-j}$ hence $V \subset B\left(x_{V}, \varepsilon\right)$. Then by (9), $\bigcup_{n=j}^{\infty}\left\{x_{V}: V \in \mathscr{Z}\left(C_{n}, n\right)\right\} \subset \bigcup_{V \in \mathscr{Z}\left(C_{j}, j\right)} B\left(x_{V}, \varepsilon\right)$. Since $\mathscr{Z}\left(C_{j}, j\right)$ is finite and $\bigcup_{n=1}^{j}\left\{x_{V}: V \in \mathscr{Z}\left(C_{n}, n\right)\right\}$ is finite,

$$
\bigcup_{n=1}^{\infty}\left\{x_{V}: V \in \mathscr{Z}\left(C_{n}, n\right)\right\}
$$

is totally bounded. For showing that $\bigcap_{n=1}^{\infty} \overline{\cup \mathscr{Z}\left(C_{n}, n\right)}$ is totally bounded, it is enough to observe that for each $n \in \mathbb{N}, \mathscr{Z}\left(C_{n}, n\right)$ is finite and that by (8) each $V \in \mathscr{Z}\left(C_{n}, n\right)$ satisfies $\operatorname{diam} V<2^{-n}$.

For each $n \in \mathbb{N}$, pick distinct $y_{n} \in S_{n}$. Then $\left\{y_{n}: n \in \mathbb{N}\right\}$ is a closed and discrete subset of $Y$, hence there exists an open and discrete family $\left\{O_{n}: n \in \mathbb{N}\right\}$ such that for each $n \in \mathbb{N}, y_{n} \in O_{n}$. For each $n \in \mathbb{N}$, let $h_{n} \in C_{p}^{*}(Y)$ be a Urysohn function satisfying $h_{n}\left(y_{n}\right)=1$ and $h_{n}\left(Y \backslash O_{n}\right)=0$. Let $\mathscr{M}$ be an uncountable almost disjoint family on $\mathbb{N}$ (i.e. for $M, N \in \mathscr{M}$ we have $M \cap N$ is finite). For $M \in \mathscr{M}$, let $f_{M}=\sum_{n \in M} h_{n}$. Then $f_{M} \in C_{p}^{*}(Y)$ and $\left\|f_{M}\right\|=1$, hence there exists $g_{M} \in C_{p}^{*}(X)$ such that $\phi\left(g_{M}\right)=f_{M}$ and $\left\|g_{M}\right\| \leq k$. Since $K$ is a nonempty compactum, there is for each $g \in C_{p}^{*}(X)$ a positive number $\delta(g)$ such that

(11) if $a, b \in B(K, \delta(g))$ with $d(a, b)<\delta(g)$, then $|g(a)-g(b)|<\frac{1}{12 k}$. 
Since $\mathscr{M}$ is uncountable we can find an uncountable subset $\mathscr{M}_{0}$ of $\mathscr{M}$ and $d>0$ such that for each $M \in \mathscr{M}_{0}, \delta\left(g_{M}\right)>d$. Let $D=B(K, d)$.

Claim 3. There is an uncountable subset $\mathscr{M}_{1}$ of $\mathscr{M}_{0}$ such that for each $M, N \in$ $\mathscr{M}_{1}$ and for each $x \in K,\left|g_{M}(x)-g_{N}(x)\right|<\frac{1}{12 k}$.

Since $C_{u}^{*}(K)$ is separable [17, Proposition 7.6.2], it contains a countable dense subset $\left\{g_{i}: i \in \mathbb{N}\right\}$. For $i \in \mathbb{N}$, let $\mathscr{M}_{0}^{i}=\left\{M \in \mathscr{M}_{0}: g_{i} \in B\left(g_{M} \mid K, \frac{1}{24 k}\right)\right\}$. Then $\mathscr{M}_{0}=\bigcup_{i=1}^{\infty} \mathscr{M}_{0}^{i}$, hence there is $i \in \mathbb{N}$ with $\mathscr{M}_{0}^{i}$ uncountable. Let $\mathscr{M}_{1}=$ $\mathscr{M}_{0}^{i}$.

By this claim, (11) and the special choice of the number $d$ we get

(12) for each $M, N \in \mathscr{M}_{1}$ and $x \in D,\left|g_{M}(x)-g_{N}(x)\right|<\frac{1}{4 k}$.

By (9) we have for each $n \in \mathbb{N}, \bigcup \mathscr{Z}\left(C_{n+1}, n+1\right) \subset \bigcup \mathscr{Z}\left(C_{n}, n\right)$. Furthermore for $V \in \mathscr{Z}\left(C_{n}, n\right), V \cap K \neq \varnothing$ and by (8) and (3), diam $V<2^{-n}$, hence there exists $n_{0} \in \mathbb{N}$ such that for $n \geq n_{0}, \cup \mathscr{Z}\left(C_{n}, n\right) \subset D$. Since $\mathscr{M}_{1}$ is uncountable and almost disjoint we can find $n \geq n_{0}$ and $M, N \in \mathscr{M}_{1}$ such that $f_{M}\left(y_{n}\right)=1$ and $f_{N}\left(y_{n}\right)=0$. For convenience let $A_{n}=\operatorname{supp}\left(y_{n}\right) \backslash \bigcup \mathscr{Z}\left(C_{n}, n\right)$, $A_{0}=\operatorname{supp}\left(y_{n}\right) \backslash \bigcup \mathscr{Z}\left(C_{0}, 0\right)$ and for $i \leq n-1$,

$$
A_{n}^{i}=\left(\operatorname{supp}\left(y_{n}\right) \backslash \bigcup \mathscr{Z}\left(C_{i+1}, i+1\right)\right) \cap \bigcup \mathscr{Z}\left(C_{i}, i\right) .
$$

We have by (9) and (10)

$$
\begin{aligned}
\sum_{a \in \operatorname{supp}\left(y_{n}\right) \backslash D}\left|\lambda_{a}\right| & \leq \sum_{a \in A_{n}}\left|\lambda_{a}\right| \leq \sum_{i=0}^{n-1} \sum_{a \in A_{n}^{i}}\left|\lambda_{a}\right|+\sum_{a \in A_{0}}\left|\lambda_{a}\right| \\
& \leq \sum_{i=0}^{n} \varepsilon_{i}=\sum_{i=0}^{n} \frac{1}{k \cdot 2^{i+3}}<\frac{1}{4 k} .
\end{aligned}
$$

Furthermore by Claim 1 and (12)

$$
\begin{aligned}
& \sum_{a \in \operatorname{supp}\left(y_{n}\right) \cap D}\left|\lambda_{a}\left(g_{M}(a)-g_{N}(a)\right)\right| \\
& \quad \leq \sum_{a \in \operatorname{supp}\left(y_{n}\right) \cap D}\left|\lambda_{a}\right| \cdot \frac{1}{4 k} \leq \frac{1}{4 k} \sum_{a \in \operatorname{supp}\left(y_{n}\right)}\left|\lambda_{a}\right| \leq \frac{1}{4 k} \cdot k=\frac{1}{4} .
\end{aligned}
$$

These two calculations give us

$$
\begin{aligned}
& \left|f_{M}\left(y_{n}\right)-f_{N}\left(y_{n}\right)\right|=\left|\phi\left(g_{M}\right)\left(y_{n}\right)-\phi\left(g_{N}\right)\left(y_{n}\right)\right| \leq \sum_{a \in \operatorname{supp}\left(y_{n}\right)}\left|\lambda_{a}\left(g_{M}(a)-g_{N}(a)\right)\right| \\
& \quad \leq \sum_{a \in \operatorname{supp}\left(y_{n}\right) \cap D}\left|\lambda_{a}\left(g_{M}(a)-g_{N}(a)\right)\right|+\sum_{a \in \operatorname{supp}\left(y_{n}\right) \backslash D}\left|\lambda_{a}\right| \cdot\left\|g_{M}-g_{N}\right\| \\
& \quad \leq \frac{1}{4}+\frac{1}{4 k} \cdot 2 k=\frac{3}{4}
\end{aligned}
$$

which is a contradiction.

Corollary 4.3. Complete metrizability is preserved by $l_{p}^{*}$-equivalence in the class of metric spaces.

In [15] it was shown that $\mathbb{Q}$ and $T$ are not $l_{p}^{*}$-equivalent, where $T$ is the space defined on p. 5. This result now also follows from Corollary 4.3. In [6] an example is given of $l_{p}$-equivalent spaces which are not $l_{p}^{*}$-equivalent. It is 
not known whether $l_{p}^{*}$-equivalent spaces are also $l_{p}$-equivalent. If this is true then Corollary 4.3 would follow from Corollary 3.4 .

\section{REMARKS}

For a space $X$, let $\mathscr{K}(X)$ be the set of compact subsets of $X$. In [8] (see also [9 and 16]), J. P. R. Christensen proved the following

Theorem 5.1. Let $X$ and $Y$ be metric spaces and let $F: \mathscr{K}(X) \rightarrow \mathscr{K}(Y)$ be a function such that

1. for every $A, B \in \mathscr{K}(X)$ with $A \subset B, F(A) \subset F(B)$ (i.e., $F$ is monotone),

2. for every $K \in \mathscr{K}(Y)$ there is $A \in \mathscr{K}(X)$ with $K \subset F(A)$ (i.e., $F(\mathscr{K}(X)$ ) is cofinal in $\mathscr{K}(Y))$.

If $X$ is separable and completely metrizable, then $Y$ is separable and completely metrizable.

This theorem leads us to an alternative proof of Theorem 3.3 provided the metric space $X$ is separable. Suppose $X$ and $Y$ are metric spaces and $\phi: C_{p}(X) \rightarrow C_{p}(Y)$ is a continuous linear surjection. Define $F: \mathscr{K}(X) \rightarrow$ $\mathscr{K}(Y)$ by

$$
F(A)=\{y \in Y: \operatorname{supp}(y) \subset A\} .
$$

By Lemma 3.1, $F$ is well defined. Obviously $F$ is monotone. To prove that $F(\mathscr{K}(X))$ is cofinal in $\mathscr{K}(Y)$, let $K \in \mathscr{K}(Y)$. For $A=\overline{\operatorname{supp} K}$, we have by Proposition 2.2, $A \in \mathscr{K}(X)$. It is easily seen that $K \subset F(A)$. We conclude that $F$ satisfies the conditions of Theorem 5.1, hence for separable completely metrizable $X$ we have $Y$ is separable and completely metrizable.

Theorem 5.1 is not valid for nonseparable $X$. Take for example $Y=\mathbb{Q}$ and $X=\bigoplus_{A \in \mathscr{A}} A$, where $\mathscr{A}=\{A \subset \mathbb{Q}: A$ is compact $\}$. Then $X$ is completely metrizable but not separable and $Y$ is separable but not completely metrizable. Define $F: \mathscr{K}(X) \rightarrow \mathscr{K}(Y)$ by $F(B)=\bigcup_{A \in \mathscr{A}}(A \cap B)$. Then $F$ satisfies the conditions in Theorem 5.1.

To give an alternative proof of Theorem 3.3 also for nonseparable spaces $X$, we have to add an extra condition on the map $F: \mathscr{K}(X) \rightarrow \mathscr{K}(Y)$, namely

3. for each locally finite open cover $\mathscr{P}$ of $X$ and each $y \in Y$, there is a neighborhood $U$ of $y$ and a finite subset $\mathscr{F}$ of $\mathscr{P}$ such that for any compact subset $K$ of $U$, there is a compact subset $L$ of $\bigcup \mathscr{F}$ such that $K \subset F(L)$.

Following the proof of Theorem 5.1 in [16] one can see that this extra property gives a generalized version of Theorem 5.1 .

Theorem 5.2. Let $X$ and $Y$ be metric spaces and let $F: \mathscr{K}(X) \rightarrow \mathscr{K}(Y)$ be a function satisfying conditions 1,2 and 3 . Then $Y$ is completely metrizable whenever $X$ is.

In case $X$ is separable, condition 3 follows from conditions 1 and 2. Since $X$ is separable, each locally finite open cover of $X$ is countable. Let $\mathscr{P}=$ $\left\{V_{n}: n \in \mathbb{N}\right\}$ be a locally finite open cover of $X$ and $y \in Y$. Let $\left\{U_{n}: n \in \mathbb{N}\right\}$ be a decreasing neighborhood base at $y$. Suppose condition 3 does not hold. Then for each $n \in \mathbb{N}$ there is a compact subset $K_{n}$ of $U_{n}$ such that for each compact subset $L$ of $\bigcup_{i=1}^{n} V_{i}, K_{n} \not \subset F(L)$. Let $K=\bigcup_{n=1}^{\infty} K_{n} \cup\{y\}$. Then $K$ is compact, hence by condition 2 there is a compact subset $L$ of $X$ such that 
$K \subset F(L)$. Since $\mathscr{P}$ is locally finite, there is $n \in \mathbb{N}$ with $L \subset \bigcup_{i=1}^{n} V_{i}$. But then $K_{n} \not \subset F(L)$ which is a contradiction.

From Lemma 2.3 in [4] it follows that the map $F: \mathscr{K}(X) \rightarrow \mathscr{K}(Y)$ constructed above from a continuous linear surjection $\phi: C_{p}(X) \rightarrow C_{p}(Y)$ satisfies condition 3, hence Theorem 5.2 gives us an alternative proof of Theorem 3.3.

This strategy seems not to be working for a continuous linear surjection $\phi: C_{p}^{*}(X) \rightarrow C_{p}^{*}(Y)$. Of course we are free to define $F: \mathscr{K}(X) \rightarrow \mathscr{K}(Y)$ by $F(A)=\{y \in Y: \operatorname{supp}(y) \subset A\}$ and by Lemma 5.4 below this function is well defined, but in general $F(\mathscr{K}(X))$ will not be cofinal in $\mathscr{K}(Y)$. The example on p. 5 gives a situation where the map $F$ does not satisfy conditions 2 and 3 .

We finish this section by discussing the following

Question. Let $X$ and $Y$ be metric spaces and $\phi: C_{p}(X) \rightarrow C_{p}(Y)$ (resp. $\left.\phi: C_{p}^{*}(X) \rightarrow C_{p}^{*}(Y)\right)$ be a continuous linear surjection. Let $\mathscr{M}$ be a Borel class of spaces. It is true that $Y \in \mathscr{M}$ whenever $X \in \mathscr{M}$ ?

Theorems 3.3 and 4.2 solve this question for the class of all absolute $G_{\delta}$ 's. We show that for the class of all compact spaces and the class of all $\sigma$-compact spaces there also is a positive answer.

Theorem 5.3. Let $X$ and $Y$ be metric spaces and let $\phi: C_{p}(X) \rightarrow C_{p}(Y)$ be a continuous linear surjection. Then

1. if $X$ is compact, then $Y$ is compact,

2. if $X$ is $\sigma$-compact, then $Y$ is $\sigma$-compact.

Proof. For $X$ compact we have by Lemma 3.1 that the set $\{y \in Y: \operatorname{supp}(y) \subset$ $X\}=Y$ is compact. For (2) let $X=\bigcup_{n=1}^{\infty} X_{n}$ with for each $n \in \mathbb{N}, X_{n} \subset X_{n+1}$ and $X_{n}$ compact. Let $Y_{n}=\left\{y \in Y: \operatorname{supp}(y) \subset X_{n}\right\}$. By Lemma 3.1 we have $Y_{n}$ is compact. Since for each $y \in Y, \operatorname{supp}(y)$ is finite we also have that $Y=\bigcup_{n=1}^{\infty} Y_{n}$.

To prove the same result for the bounded case we need the following

Lemma 5.4. Let $X$ and $Y$ be metric spaces and let $\phi: C_{p}^{*}(X) \rightarrow C_{p}^{*}(Y)$ be a continuous linear surjection. Then for each compact $K \subset X$, the set $L=\{y \in$ $Y: \operatorname{supp}(y) \subset K\}$ is compact.

Proof. By Proposition 2.3, supp is LSC, hence $L$ is closed in $Y$. For $f \in$ $C_{p}^{*}(K)$, let $\tilde{f} \in C_{p}^{*}(X)$ be an extension of $f$. Define $\theta: C_{p}^{*}(K) \rightarrow C_{p}^{*}(L)$ by $\theta(f)=\phi(\tilde{f}) \mid L$. If $g \in C_{p}^{*}(X)$ is another extension of $f$, then $\tilde{f}$ and $g$ coincide on $\operatorname{supp} L$, hence by Corollary $2.1 \phi(\tilde{f})=\phi(g)$ on $L$. This implies that $\theta$ is well defined. It follows that $\theta$ is a continuous linear function. By the Closed Graph Theorem, $\theta$ considered as a function from $C_{u}^{*}(K)$ to $C_{u}^{*}(L)$ is also continuous. We claim that $\theta$ is surjective. Let $g \in C_{p}^{*}(L)$ and let $\tilde{g} \in C_{p}^{*}(Y)$ be an extension of $g$. Since $\phi$ is surjective, there is $h \in C_{p}^{*}(X)$ with $\phi(h)=\tilde{g}$. Let $f=h \mid K$. Since $h$ extends $f, \theta(f)=g$, so $\theta$ is a surjection. By [17, Proposition 7.6.2] we have for a space $Z$ that $C_{u}^{*}(Z)$ is separable if and only if $Z$ is compact and metrizable. This implies that $C_{u}^{*}(K)$ is separable and hence $C_{u}^{*}(L)$ is separable. So $L$ is compact.

Using Lemma 5.4 instead of Lemma 3.1 we also obtain 
Theorem 5.5. Let $X$ and $Y$ be metric spaces and let $\phi: C_{p}^{*}(X) \rightarrow C_{p}^{*}(Y)$ be a continuous linear surjection. Then

1. if $X$ is compact, then $Y$ is compact, and

2. if $X$ is $\sigma$-compact, then $Y$ is $\sigma$-compact.

\section{REFERENCES}

1. A. V. Arhangel'skiī, On linear homeomorphisms of function spaces, Soviet Math. Dokl. 25 (1982), 852-855.

2. ___, Problems in $C_{p}$-theory, Open Problems in Topology, (J. van Mill and G. M. Reed, eds.), North-Holland, Amsterdam, 1990, pp. 601-615.

3. J. Baars and J. de Groot, An isomorphical classification of function spaces of zero-dimensional locally compact separable metric spaces, Comment. Math. Univ. Carolin. 29 (1988), 577-595.

4. __ On the $l_{p}$-equivalence of metric spaces, Fund. Math. 137 (1991), 25-43.

5. J. Baars, J. de Groot, and J. van Mill, A theorem on function spaces, Proc. Amer. Math. Soc. 105 (1989), 1020-1024.

6. J. Baars, J. de Groot, J. van Mill, and J. Pelant, An example of $l_{p}$-equivalent spaces which are not $l_{p}^{*}$-equivalent, Proc. Amer. Math. Soc. (to appear).

7. __. On topological and linear homeomorphisms of certain function spaces, Topology Appl. 32 (1989), 267-277.

8. J. P. R. Christensen, Necessary and sufficient conditions for the measurability of certain sets of closed subsets, Math. Ann. 200 (1973), 189-193.

9. _ Topology and Borel structure, Math. Studies, North-Holland, Amsterdam, 1974.

10. T. Dobrowolski, S. P. Gul'ko, and J. Mogilski, Function spaces homeomorphic to the countable product of $l_{f}^{2}$, Topology Appl. 34 (1990), 153-160.

11. I. Gohberg and S. Goldberg, Basic operator theory, Birkhäuser, 1981.

12. K. Kunen, Set theory, an introduction to independence proofs, Studies in Logic, vol. 102, North-Holland, Amsterdam, 1980.

13. J. van Mill, Infinite dimensional topology: prerequisites and introduction, North-Holland, Amsterdam, 1989.

14. D. Pavlovskii, On spaces of continuous functions, Soviet Math. Dokl. 22 (1980), 34-37.

15. J. Pelant, A remark on spaces of bounded continuous functions, Indag. Math. 91 (1988), 335-338.

16. J. Saint Raymond, Caracterisation d'espaces polonais, Seminaire Choquet (initiation á l'analyse), $11^{e}-12^{e}$ annees (1971-73), Secrétariat Math., Paris, 1970.

17. Z. Semadeni, Banach spaces of continuous functions, PWN, Warsaw, 1971.

18. V. V. Uspenskiī, On the topology of a free locally convex space, Soviet Math. Dokl. 27 (1983), 781-785.

Faculteit Wiskunde en Informatica, Universiteit van Amsterdam, Plantage MuiderGRACHT 24, 1018 TV AMSTERDAM, The NeTherLandS

Faculteit Wiskunde en Informatica, Vrije Universiteit, De BoelelaAn 1081, 1081 HV AMSTERDAM, The Netherlands

Matematický Ústav, Československá AKademie Věd, Žitná 25, 11567 Praha, ČeskoloVENSKO 